IAU Colloquium 164: Radio Emission from Galactic and Extragalactic Compact Sources

ASP Conference Series, Vol. 144, 1998

J. A. Zensus, G. B. Taylor, \& J. M. Wrobel (eds.)

\title{
Proper Motions and Jets in NGC 4258: Further Evidence for a Sub-Parsec Nuclear Disk
}

\author{
J. R. Herrnstein ${ }^{1}$ \\ Harvard-Smithsonian Center for Astrophysics, Cambridge, MA, USA.
}

Abstract. The evidence for a subparsec-scale disk in the nucleus of NGC 4258 is reviewed in the context of multi-epoch VLBA observations. The detection of jet continuum emission and of maser proper motions in these data bolster the case for a nuclear disk.

\section{Introduction}

The nuclear megamaser in NGC 4258 is thought to trace a thin, nearly edge-on, sub-parsec disk in Keplerian rotation around a supermassive compact object (Watson \& Wallin 1994; Greenhill et al. 1995a; Miyoshi et al. 1995; Moran et al. 1995). We have continued to monitor the maser in NGC 4258 with the VLBA of the NRAO, and in this contribution I will re-examine the disk interpretation in light of these new data. The multi-epoch VLBA data allow for new and powerful tests of the disk paradigm, and these will be discussed in detail.

\section{Positions, Velocities, and Accelerations}

Figure 1 summarizes the NGC 4258 VLBA data. The data taken in April 1994 were originally presented in Miyoshi et al. (1995). The upper 2 panels of the figure concern the high-velocity maser emission in NGC 4258. These features (discovered by Nakai, Inoue, \& Miyoshi in 1993) are symmetrically offset 1000 $\mathrm{km} \mathrm{s}^{-1}$ on either side of the central maser emission at the systemic velocity of the galaxy. The upper-left panel shows line-of-sight (LOS) velocity versus impact parameter for the high-velocity masers in each epoch, along with the best-fitting Keplerian rotation curves. The striking $1 / \sqrt{r}$ rotation law originally reported in Miyoshi et al. (1995) persists in the later epochs. The simplest explanation for the precision of this rotation curve is that the high-velocity masers lie along a single diameter of a system in Keplerian rotation. The upper right panel of Figure 1 shows LOS velocity versus time for the high-velocity feature with LSR velocity $1306 \mathrm{~km} \mathrm{~s}^{-1}$. This feature, which is $838 \mathrm{~km} \mathrm{~s}^{-1}$ and $0.2 \mathrm{pc}$ from the center of the putative disk, is persistently strong and isolated and is easily tracked between epochs. The acceleration of the feature is constrained by these data to be less than about $0.1 \mathrm{~km} \mathrm{~s}^{-1} \mathrm{yr}^{-1}$ suggesting that it lies within about $2^{\circ}$ of the disk midline, the diameter defined by the intersection of the disk with the plane of the sky. The Keplerian rotation curve then implies all the high-velocity masers are distributed along this midline. This is consistent with the fact that the longest coherent velocity gain paths are along the midline of the disk.

The bottom two panels of Figure 1 focus on the systemic maser emission. The left-hand panel shows that the velocity gradient originally noted by Greenhill et al. (1995a) persists in the VLBA data, and is approximately constant at

${ }^{1}$ Present address: National Radio Astronomy Observatory, Socorro, NM, USA. 

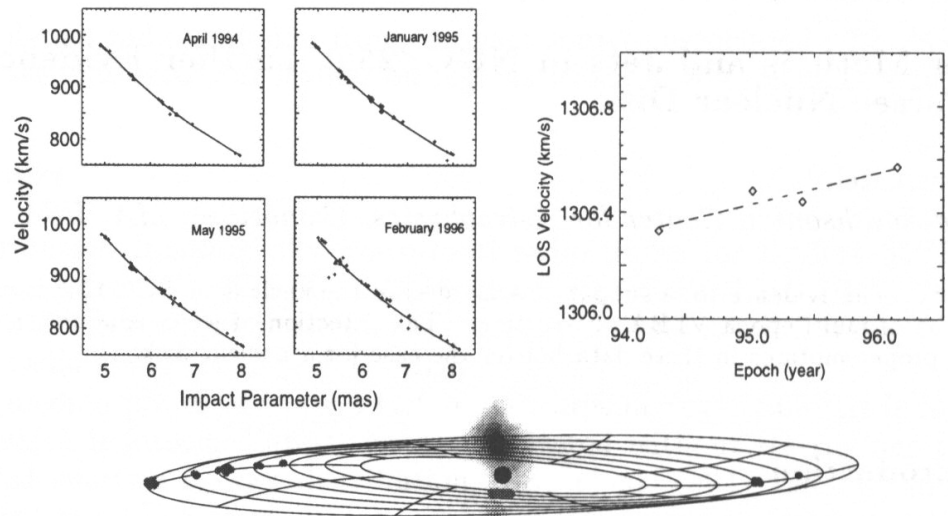

$0.4 \mathrm{ly}$
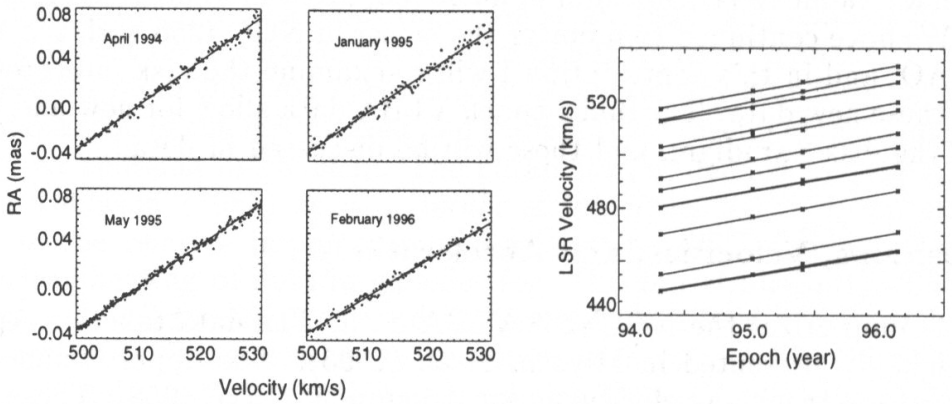

Figure 1. Position, velocity, and LOS accelerations for the masers in NGC 4258; see text for details.

$290 \mathrm{~km} \mathrm{~s}^{-1} \mathrm{mas}^{-1}$ over the 2 years spanned by the observations. While solid body rotation could lead to such a position velocity gradient, the Keplerian rotation curve of the high-velocity masers suggests that the systemic masers are in fact confined to a narrow annulus in a differentially rotating disk. The lower right-hand panel of the figure shows LOS velocity versus time for 13 systemic features that can be confidently tracked across all the VLBA observations. The average acceleration is about $9 \mathrm{~km} \mathrm{~s}^{-1} \mathrm{yr}^{-1}$ and is consistent with more extensive single-dish monitoring programs of Greenhill et al. (1995b) and Nakai et al. (1995). These accelerations are consistent with the expected centripetal accelerations along the near edge of the NGC 4258 disk for a reasonable distance to the galaxy (cf. Watson \& Wallin 1994), and the small scatter in the accelerations is further proof that they are confined to a narrow range in radius.

The central panel of Figure 1 shows the best-fitting disk model generated using a formal global $\chi^{2}$ analysis incorporating the VLBA positions and velocities (Herrnstein 1997). All of the disk characteristics inferred from the foregoing analysis are readily apparent, and the excellent fit to the data (reduced $\chi^{2}$ of 1.6 ) is strong evidence for a single disk structure in the nucleus of NGC 4258 . 


\section{Continuum Emission}

The central panel of Figure 1 also shows $22 \mathrm{GHz}$ continuum emission detected in three of the four VLBA epochs. The emission traces a subparsec-scale jet directed along the rotation axis of the disk. The northern component of the jet is about 4000 Schwarzschild radii from the disk center. The southern feature is twice as far from the center, and about one quarter as bright as the north. The continuum emission in NGC 4258 is discussed in the context of theories of subparsec-scale jets and maser amplification elsewhere (Herrnstein et al. 1997). Here, I point out that the excellent alignment between the jet and disk axes is further evidence for the disk geometry. The alignment between the projected axes is readily apparent in Figure 1. The global disk models also indicate that the disk is tipped down from edge-on by about $8^{\circ}$ and that the southern jet should lie behind the near edge of the disk. This too is indirectly confirmed by the continuum observations. Optical depths of $2-3$ are expected in the partially and fully ionized gas along the surface of the disk (Herrnstein, Greenhill, \& Moran 1996), and this can account for the relative faintness of the southern jet emission. In addition, the brightness and time-variability of the systemic masers implicate amplification of background radiation, and the southern jet emission is a plausible source.

\section{Proper Motions}

While the red- and blue-shifted high-velocity features should remain fixed in right ascension (RA) and declination (dec) with respect to one another, the $1000 \mathrm{~km} \mathrm{~s}^{-1}$ transverse velocities of the systemic features should result in proper motions of about $30 \mu \mathrm{as} \mathrm{yr}^{-1}$, assuming a distance of $6.5 \mathrm{Mpc}$ to NGC 4258 . Such motions should be easily detectable in the present data.
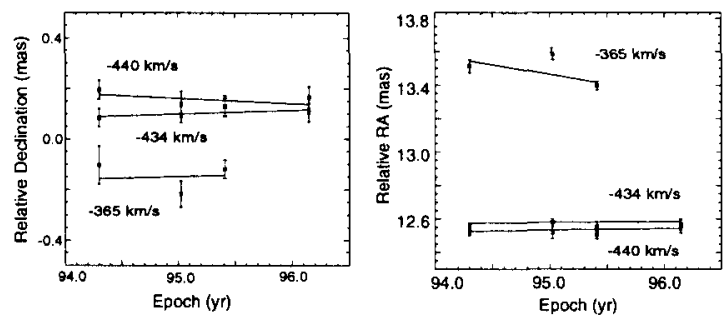

Figure 2. RA (upper) and $\operatorname{dec}$ (lower) versus time for the blue-shifted features with respect to the red-shifted feature at $1306 \mathrm{~km} \mathrm{~s}^{-1}$.

Figure 2 shows the time evolution of the RA and dec of the three blue-shifted features with respect to the red-shifted feature at $1306 \mathrm{~km} \mathrm{~s}^{-1}$. We detect no relative motion between the red-and blue-shifted masers, thus corroborating the disk model. The relatively poor signal-to-noise to the blue-shifted features results

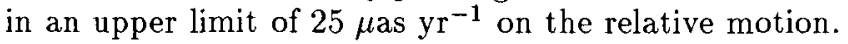

Figure 3 shows proper motion tracks for the 13 systemic features that can be unambiguously tracked in the VLBA data. We have successfully detected proper motions in the systemic masers in NGC 4258. For a reasonable distance 


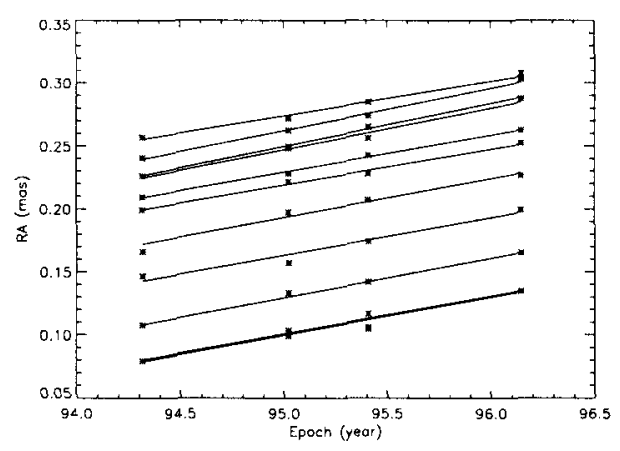

Figure 3. RA versus time for 13 systemic features.

to $N G C 4258$ of $6.5 \mathrm{Mpc}$, the average motion of $30 \mu a s \mathrm{yr}^{-1}$ corresponds to a transverse velocity of about $1000 \mathrm{~km} \mathrm{~s}^{-1}$, which is in excellent agreement with the disk models. We note that the LOS accelerations and proper motions together can be used to derive a purely geometrical distance to NGC 4258 that is largely free of systematic uncertainties (Herrnstein 1997). The results of such an analysis will be presented elsewhere.

\section{Conclusions}

The four VLBA epochs discussed here provide additional support for the disk interpretation of the masers in NGC 4258. The detection of continuum jet emission confirms the 3 -dimensional orientation of the disk rotation axis. Proper motion observations demonstrate that the systemic features possess transverse velocities of $\sim 1000 \mathrm{~km} \mathrm{~s}^{-1}$ while the high-velocity features have negligible transverse motions, in good agreement with the disk models.

Acknowledgments. The National Radio Astronomy Observatory is a facility of the National Science Foundation, operated under a cooperative agreement by Associated Universities, Inc.

\section{References}

Greenhill, L. J., et al. 1995a. $A p J, 440,619-627$.

Greenhill, L. J., et al. 1995b. A 8 A, 304, 21-33.

Herrnstein, J. R. 1997. Ph.D. Thesis, Harvard University.

Herrnstein, J. R., et al. 1997. ApJ, 475, L17-20.

Herrnstein, J. R, Greenhill, L. J., \& Moran, J. M. 1996. ApJ, 468, L17-20.

Miyoshi, M., et al. 1995. Nature, 373, 127-129.

Moran, J. M., et al. 1995. Proc. Natl. Acad. Sci. USA, 92, 11427-11437.

Nakai, N., et al. 1995. PASJ, 47, 771-799.

Nakai, N., Inoue, M., \& Miyoshi, M. 1993. Nature, 361, 45-47.

Watson, W. D., \& Wallin, B. K. 1994. ApJ, 432, L35-38. 